\title{
Proposed New Method to Measure Common-Mode Current on High Frequency Circuits
}

\section{Mohmed AO*}

Head of Department, Engineering Higher Institute of General Vocational, Ajdabia, Libya

\begin{abstract}
It goes without saying that electromagnetic compatibility (EMC) is very significant in our life. Therefore, measuring common-mode current will estimate how much electromagnetic waves are emitted from the specific device. This paper has reviewed two methods to measure the common-mode current (CMC), these methods are very useful help to comprehend the experiments of standard (EMC) tests. These reviewed methods have used spectrum analyzer in order to show the result of the measurements unlike the proposed method in this paper. Whereas, a steps of designing and calibrated a tool that can measure the magnitude value of the $(\mathrm{CMC})$ are covered in this article. The different between the first and second reviewed method for measurement of (CMC) is that both of them use spectrum analyzer but, the proposed methods uses (LCD display) so it makes the designing tool would be portable to do the measurements, so no need to do it in the lab.
\end{abstract}

Keywords: EMC; Common-mode; Spectrum; LCD; Microcontroller

\section{Introduction}

In the beginning of improvement era of electrical devices a lot of considerations were ignored in the electrical industrial about safety and environment. Besides, in last 20 years, many tragic actual events were happened without having a good cause to figure out what has been gone wrong to get a failure in operation properly such as: an airplane fall down causing the death of 229 people due to engine miss controlled in control panel system and many other alike unexplained tragic events so that made the scientists instigate and study why did that happen [1]. After having a lot of experimental and getting a lot of results analyzed, scientists showed these were happened due to the electromagnetic wave interference. Moreover, that is led to have the Electromagnetic compatibility (EMC's) standards to ensure the human's and other electric devices' safety. Furthermore, (EMC) has four main tests which are radiation emission, tradition immunity, conducted emission and conducted immunity. However, the hardest test to pass is the radiation emissions where the device is tested by measuring how many electrical and magnetic waves are emitted by the equipment under test. Common-mode current is a main source of Electromagnetic radiation interference [2].

Thus, measurement of common-mode current (CMC) is very important to estimate the electromagnetic radiated waves. This paper reviews some used technics to measure CMC and a proposed a new portable tools to measure the magnitude of (CMC).

\section{Related Work}

This paper reviews to methods to measure the common mode current by using spectrum analyzer.

\section{Measurement system for common mode current using (LISN)}

This study a gives a new method to measure CMC by using spectrum analyzer and coaxial cables and line Impedance stabilization network (LISN) [2].

The probe plays a significant part in this measurement method for (CMC) and (DMC) radiated wave's interference. Removing the interference frequency magnitude of the phase line, the neutral line and $(\mathrm{CMC})$, correspondingly. The experiment results can be used to measure the $(\mathrm{DMC})$ interference magnitude.
Set off for measuring the noise caused by equipment under test (EUT) on the power line is shown in Figure 1 [2].

Decoupling the (EUT) from the power grid, a line impedance stabilization network (LISN) is used. Current probes are used to measure conducted noise [2]. Using toroid from of magnetic probe that allows having good wiring arrangements that can be utilized to measure the (CMC) and (DMC) interferences. Figure 1 shows the wire arrangements for (DMC) and (CMC) noise measurements, consecutively [2]. Spectrum analyzer is used to show the measurements of the output port of the probe. After calibration producers and implementing the software are done in the article [2]. Then, the measurements of (DMC) is done by using known source where (DMC) is $\vec{I}_{D M}$ and it is defined by the current signals $\vec{I}_{L}, \vec{I}_{N}$ and $\vec{I}_{C M}$.

$$
\left|\vec{I}_{D M}\right|=\sqrt{\frac{\left(\left|\vec{I}_{L}\right|^{2}\right)+\left(\left|\vec{I}_{N}\right|^{2}\right)-2\left(\left|\vec{I}_{C M}\right|^{2}\right)}{2}}
$$

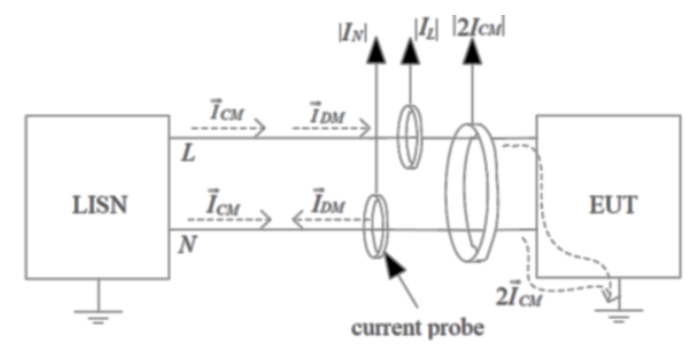

Figure 1: Measurement arrangement.

${ }^{*}$ Corresponding author: Mohmed AO, Head of Department, Engineering Higher Institute of General Vocational, Ajdabia, Libya, Tel: 00218928030406; E-mail: Ahmedalhasady911@gmail.com

Received June 21, 2018; Accepted July 28, 2018; Published August 04, 2018

Citation: Mohmed AO (2018) Proposed New Method to Measure CommonMode Current on High Frequency Circuits. J Electr Electron Syst 7: 266. doi: 10.4172/2332-0796.1000266

Copyright: (C) 2018 Mohmed AO. This is an open-access article distributed under the terms of the Creative Commons Attribution License, which permits unrestricted use, distribution, and reproduction in any medium, provided the original author and source are credited. 
Citation: Mohmed AO (2018) Proposed New Method to Measure Common-Mode Current on High Frequency Circuits. J Electr Electron Syst 7: 266. doi: $10.4172 / 2332-0796.1000266$

Page 2 of 3

Where the $\vec{I}_{D M}$ from Equation 1 current amplitude can be calculated.

\section{Non-commercially common-mode current}

Commercial current probes have been offered on marketplace for many years. Thus, current probe can be made in our own. Therefore, current probes help the researches to comprehend the current probe methods of measurements. A straightforward method to make current probe is by using toroid shape of ferrite core. By making number of turns around the ferrite, the ferrite acts identical like a secondary transformer [3]. The basic prototype of current probe is showed in Figure 2 .

The steps for making our own current probes and characterizing its transfer impedance are offered in Figure 3, are presented two of lab -made current probes [3].

The first one on the right side, electrical wire is used and the wire is soldered to the BNC connector and, coaxial cable is used instead the other one [3]. Measuring common-mode current (CMC) in amps with the network analyzer requires known value for transfer impedance of the ferrite core in fixed frequency.

\section{Proposed Mothed to Measure Common Mode Current}

This paper proposes a new way to measure common-mode current by designing a prototype of tool that illustrates the magnitude value of the (CMC) on (LCD monitor) at fixed frequency that is done in many stages, apparatuses' calibration, calculation the transfer impedance of the used ferrite core and, microcontroller calibration.

\section{Apparatuses' calibration}

In order to design a prototype of a portable device to measure the

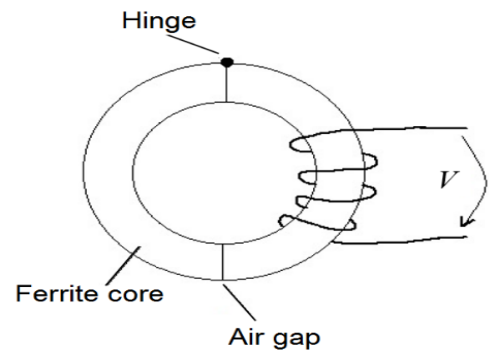

Figure 2: Basic assembly prototype of current probe.

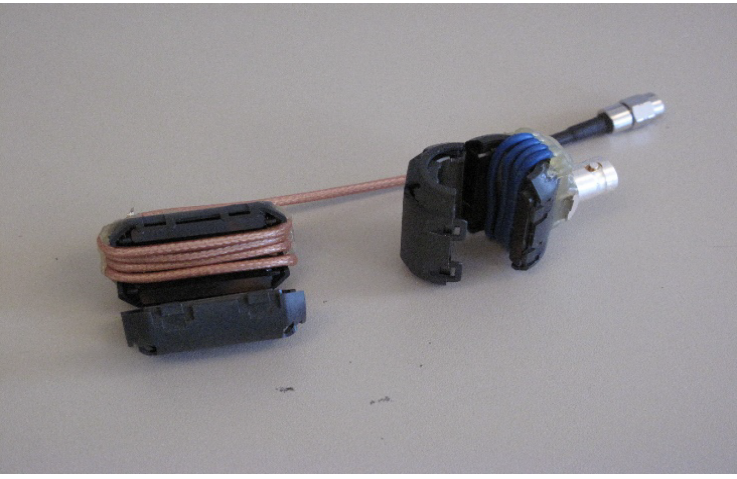

Figure 3: Lab-made current probes. magnitude common-mode current many used apparatuses have to be a calibrated.

In first stage the all considerations will be first on the apparatuses that will be used. The apparatuses are function generator, oscilloscope and the coaxial cables with two wire ends. Calibrating the signal generator will be done by using calibrated oscilloscope and, Calibrating the cables will be done by using calibrated signal generator and oscilloscope.

Transfer impedance calculations: Normal size of ferrite core will be used to form a tool to measure (CMC) and in order to do that is to set the frequency on few hundreds megahertz. Then, it will be done by making a single wire with known AC Sin wave signal voltage $V i$ flow into wire with known value load resistor RL as showing in Figure 4.

First the voltage on the resistor is measured then the current flow through the wire will be known by using Ohm's Law:

$$
I_{i}=\frac{V_{i}}{R_{L}}
$$

After current through the wire is known, then the voltage induced through the ferrite will be known by using the oscilloscope. The impedance of the ferrite core will be calculated by using Equation 3:

$$
Z_{T}=\frac{V_{i}}{I_{i}}
$$

\section{Microcontroller calibration}

To show the magnitude value of the CMC microcontroller must be used such as Interface Controller (PIC) (18F4450) and that is done by using (Enhanced 40 pins PIC Start-Up Kit) Figure 5, Or Arduino board with liquid crystal display LCD monitor to show the measurements Figure 5.

However that would not be easy because the AC voltage signal would be very small and with high frequency that would be hard to rectifier

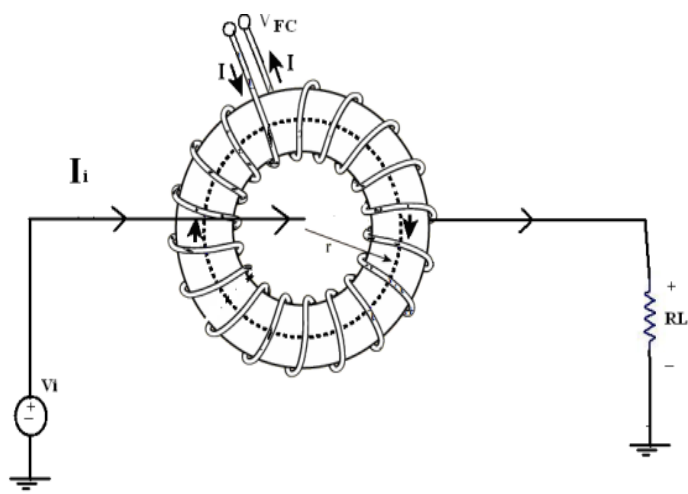

Figure 4: circuit of calculation the transfer impedance.

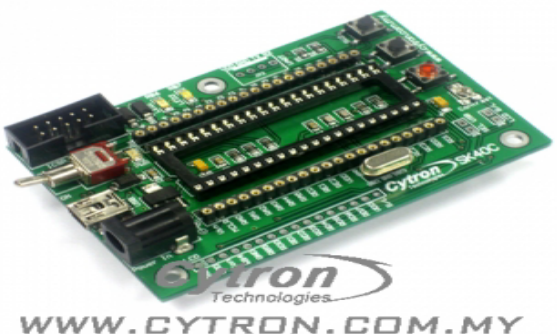

Figure 5: Enhanced 40 pins PIC Start-Up Kit. 
to convert to DC signal to make it the input of the microcontroller. Therefore envelope detector (IC LTC 5532) is advised to use to convert the AC to DC signal [4].

The stage of converting the AC to DC signal has to be calibrated to get the calibrated factor $\mathrm{CF}$ in order to put it in the programed code that will be uploaded to the used microcontroller by using Equation 4:

$$
I_{C}=\left(\frac{V_{F C} * C F}{Z_{T}}\right)
$$

Where $I_{C}$ is the common mode current and, $V_{F C}$ is the voltage induced from the used ferrite core and $Z_{T}$ is the calculated transfer impedance at fixed frequency. So, the calibration of the AC to DC signal converter is to set the $\mathrm{CF}$ by increasing and reading the input voltage of the converting IC and read the output of the converting IC and set a equation that equation would be linear equation that linear equation is the $\mathrm{CF}$.

\section{Discussion and Conclusion}

This paper reviewed two methods to measure the common-mode current (CMC). These methods help to understand the challenges of standard EMC tests. These reviewed methods have used spectrum analyzer in order to show the result of the measurements unlike the proposed method in this paper. Whereas, a steps of designing and calibrated a tool that can measure the magnitude value of the (CMC) and presents it on proposed (LCD display) by using programed microcontroller. The different between the reviewed method for measurement of (CMC) is the reviewed methods uses spectrum analyzer so, that the measurement set up only will be taking place just in the lab but the proposed methods uses (LCD display) so it makes the designing tool would be portable to do the measurements, so no need to do it in the lab.

\section{Acknowledgment}

In preparing this paper, the author was in contact with many people, academicians, researchers and practitioners who contributed immensely towards his understanding and thoughts. The author thanks Higher Institute of General Vocational, Ajdabia, Libya for financially supporting this work.

\section{References}

1. Ladkin PB, Schepper W (2000) EMI, TWA 800 and Swissair 111. Semantic Scholar.

2. Feng Q, Liao C, Xiong XZ (2014) A Novel Measurement System for the Common-Mode- and Differential-Mode-Conducted Electromagnetic Interference. Prog Electromagn Res Lett 48: 75-81.

3. Wyatt K (2012) The HF Current Probe: Theory and Application.

4. http://www.analog.com/media/en/technical-documentation/data-sheets/5532f.pdf 